\title{
The Asymptotic Expansion of the Meijer $G$-Function*
}

\author{
By Jerry L. Fields
}

\begin{abstract}
Gamma function identities are integrated to expand the Meijer $G$-function in a basic set of functions, each of which is simply characterized asymptotically.
\end{abstract}

1. Introduction and Notation. In this exposition, we derive the asymptotic expansion of the Meijer $G$-function for large values of the variable. Although these results can be found in various places in the literature, e.g., Meijer's original papers [1], or their collection by Luke [2], they are usually obscured by a maze of special notation and the presence of a large number of results which are only of secondary interest. The following derivation seems more direct.

Throughout this work, we assume that the integers $p, q, m, n$ and parameters $a_{i}, b_{i}$ satisfy the hypothesis,

$$
\begin{aligned}
& 0 \leqq m \leqq q, 0 \leqq n \leqq p, \\
& a_{j}-b_{k} \neq \text { a positive integer } ; j=1, \cdots, p ; k=1, \cdots, q, \\
& a_{i}-a_{k} \neq \text { an integer; } j, k=1, \cdots, p ; j \neq k .
\end{aligned}
$$

Extensive use will be made of the notations,

$$
\begin{aligned}
\Gamma_{n}\left(c_{P}-t\right) & =\prod_{k=n+1}^{p} \Gamma\left(c_{k}-t\right), \quad \Gamma\left(c_{M}-t\right)=\Gamma_{0}\left(c_{M}-t\right), \\
{ }_{p} F_{Q}\left(\begin{array}{l|l}
a_{P} & w \\
b_{Q} & w
\end{array}\right) & =\sum_{k=0}^{\infty} \frac{\Gamma\left(a_{P}+k\right) \Gamma\left(b_{Q}\right)}{\Gamma\left(b_{Q}+k\right) \Gamma\left(a_{P}\right)} \cdot \frac{w^{k}}{k !} .
\end{aligned}
$$

The Meijer $G$-function is then defined by

$$
\begin{aligned}
G_{p, q}^{m, n}(z) & =G_{p, q}^{m, n}\left(z \mid \begin{array}{c}
a_{P} \\
b_{Q}
\end{array}\right)=G_{p, q}^{m, n}\left(z \mid \begin{array}{l}
a_{1}, \cdots, a_{p} \\
b_{1}, \cdots, b_{q}
\end{array}\right) \\
& =\frac{1}{2 \pi i} \int_{L} \frac{\Gamma\left(b_{M}-t\right) \Gamma\left(1-a_{N}+t\right) z^{t}}{\Gamma_{m}\left(1-b_{Q}+t\right) \Gamma_{n}\left(a_{P}-t\right)} d t,
\end{aligned}
$$

where $L$ is an upward oriented loop contour which separates the poles of $\Gamma\left(b_{M}-t\right)$ from those of $\Gamma\left(1-a_{N}+t\right)$ and which begins and ends at $+\infty\left(L=L_{+}\right)$or $-\infty$

Received November 20, 1971.

AMS 1970 subject classifications. Primary 33A35; Secondary 33A02.

Key words and phrases. Meijer $G$-functions, asymptotic expansions, linear differential equations, irregular singular points.

* This work was initiated in 1968-69 while the author was a visiting professor at the National Tsing Hua University, Taiwan, Republic of China, and completed under grant NRC A 7549 of the National Research Council of Canada. 
$\left(L=L_{-}\right)$. A simple computation shows that $G_{p, q}^{m, n}(z)$ satisfies the linear differential equation

$$
\left\{\prod_{i=1}^{a}\left(\delta-b_{j}\right)+(-1)^{p+1-m-n} z \prod_{j=1}^{p}\left(\delta+1-a_{j}\right)\right\} y(z)=0, \quad \delta=z \frac{d}{d z},
$$

of order $\max (p, q)$.

If $q \leqq p, z=\infty$ is a regular singular point of (1.4). The behaviour of $G_{p, \alpha}^{m, n}(z)$ for $z$ large then follows from the residue calculus result, $L=L_{-}$,

THEOREM 1. Under the conditions of (1.1),

$$
\begin{aligned}
& G_{p, q}^{m, n}(z)=\sum_{j=1}^{n} \frac{\Gamma^{*}\left(a_{j}-a_{N}\right) \Gamma\left(1+b_{M}-a_{j}\right)}{\Gamma_{n}\left(1+a_{P}-a_{i}\right) \Gamma_{m}\left(a_{i}-b_{Q}\right)} z^{-1+a_{j}} \\
& \times_{q+1} F_{p}\left(\begin{array}{r}
1,1+b_{Q}-a_{i} \\
1+a_{P}-a_{j}
\end{array} \mid \frac{(-1)^{a-m-n}}{z}\right), \\
& q<p \text { or } q=p \text { and }|z|>1,
\end{aligned}
$$

where

$$
\Gamma^{*}\left(a_{i}-a_{N}\right)=\prod_{k=1 ; k \neq i}^{n} \Gamma\left(a_{i}-a_{k}\right) .
$$

If $n=0,(1.5)$ reduces to $G_{p, q}^{m, 0}(z) \equiv 0$.

If $q>p, z=\infty$ is an irregular singular point of (1.4), and the analysis is more involved. For convenience, we set

$$
\nu=q-p \geqq 1, \quad \mu=q-m-n .
$$

A special case of the above, $m=q$ and $n=0$ or 1, was treated by Barnes [3], who obtained

THEOREM 2 (BARNES). Under the conditions of (1.1), $\nu \geqq 1$,

$$
\begin{aligned}
& L_{j}(w) \equiv G_{p, Q}^{a, 1}\left(w \mid \begin{array}{l}
a_{j}, a_{1}, \cdots, a_{i-1}, a_{i+1}, \cdots, a_{p} \\
b_{1}, \cdots, b_{q}
\end{array}\right), \quad j=1, \cdots, p, \\
& \sim \frac{\Gamma\left(1+b_{Q}-a_{j}\right)}{\Gamma\left(1+a_{P}-a_{j}\right)} w^{-1+a_{i}}{ }_{q+1} F_{p}\left(\begin{array}{r}
1,1+b_{Q}-a_{j} \mid \\
1+a_{P}-a_{i}
\end{array} \mid \frac{-1}{w}\right), \\
& w \rightarrow \infty, \quad|\arg w|<\pi(\nu / 2+1),
\end{aligned}
$$

and

$$
\begin{aligned}
& G(w) \equiv G_{p, a}^{a, 0}\left(w \mid \begin{array}{l}
a_{1}, \cdots, a_{p} \\
b_{1}, \cdots, b_{q}
\end{array}\right) \\
& \sim\left(\frac{(2 \pi)^{\nu-1}}{\nu}\right)^{1 / 2} \exp \left(-\nu w^{1 / \nu}\right) \sum_{i=0}^{\infty} K_{i} w^{\gamma-i / \nu}, \\
& w \rightarrow \infty, \quad|\arg w|<\pi(\nu+\min (1, \nu / 2)),
\end{aligned}
$$

where 


$$
\begin{aligned}
\nu \gamma & =\frac{1-\nu}{2}+B_{1}-A_{1}, \quad K_{0}=1, \\
K_{1} & =A_{2}-B_{2}+\frac{\left(B_{1}-A_{1}\right)}{2 \nu}\left[\nu\left(A_{1}+B_{1}\right)+A_{1}-B_{1}\right]+\frac{1-\nu^{2}}{24 \nu}, \\
\prod_{i=1}^{p}\left(x+a_{j}\right) & =\sum_{j=0}^{p} A_{j} x^{p-i}, \quad \prod_{i=1}^{q}\left(x+b_{j}\right)=\sum_{i=0}^{q} B_{j} x^{q-i},
\end{aligned}
$$

and the remaining $K_{j}$ are polynomials in $A_{i}, B_{j}$ independent of $w$.

When $\nu \geqq 1$, the contour $L$ of (1.3) is equal to $L_{+}$. In particular, if $m=0, G_{p, q}^{0, n}(z) \equiv 0$. A straightforward computation shows that $L_{i}\left(z e^{i \pi(\mu+1-2 r)}\right)$ and $G\left(z e^{i \pi(\mu-2 s)}\right)$ are also solutions of (1.4), $r, s$ arbitrary integers. For a given value of $\arg z$, there exists at least one pair of integers $(r, s)$ such that

$$
\begin{aligned}
|\arg z+\pi(\mu+1-2 r)| & <\pi(\nu / 2+1), \\
|\arg z+\pi(\mu+2-2 s-2 h)| & <\pi(\nu+\min (1, \nu / 2)), \quad h=1, \cdots, \nu .
\end{aligned}
$$

It then follows from Theorem 2 , that in a suitable sector, the $q$ functions $L_{j}\left(z e^{i \pi(\mu+1-2 r)}\right), j=1, \cdots, p, G\left(z e^{i \pi(\mu+2-2 s-2 h)}\right), h=1, \cdots, \nu$, form a basis of solutions for (1.4).

THEOREM 3 (MEIJER). Under the conditions of (1.1), $\nu \geqq 1$, if the sector

$$
\begin{array}{cl}
S_{r, s}: & \pi\left(\nu-\mu-2+\max \left[2 r-\frac{3 \nu}{2}, 2 s-\min \left(1, \frac{\nu}{2}\right)\right]\right)<\arg z \\
& \arg z<\pi\left(\frac{\nu}{2}-\mu+\min \left[2 r, \frac{\nu}{2}+2 s+\min \left(1, \frac{\nu}{2}\right)\right]\right)
\end{array}
$$

is not empty, then there exist constants $C_{j}(r, s), D_{j}(r, s)$ such that

$$
G_{p, q}^{m, n}(z)=\sum_{j=1}^{p} C_{j}(r, s) L_{j}\left(z e^{i \pi(\mu+1-2 r)}\right)+\sum_{h=1}^{\nu} D_{h}(r, s) G\left(z e^{i \pi(\mu+2-2 s-2 h)}\right) .
$$

Equation (1.12) will be referred to as the $(r, s)$ expansion for $G_{p, q}^{m, n}(z)$.

Once the values of $C_{j}(r, s), D_{h}(r, s)$ have been determined for $z \in S_{r, s},(1.12)$ remains valid for all values of $\arg z$. However, it is useful for the asymptotic evaluation of $G_{p, q}^{m, n}(z)$ only when the arguments of the $L_{i}$ and $G$ functions which are actually present satisfy the argument restrictions of (1.8) and (1.9), respectively.

Thus, the only practical difficulty in using Theorem 3 to determine the behaviour of $G_{p, q}^{m, n}(z)$ for $z$ large lies in the determination of $C_{j}(r, s), D_{h}(r, s)$ for any given value of $\arg z$. This problem is discussed in Section 2.

If the complex conjugate of the $(r, s)$ expansion, (1.12), is taken, treating $z, a_{i}, b_{i}$ as real, one obtains the $(\mu+1-r, \mu+1-\nu-s)$ expansion. This is particularly useful when $\nu=1$ or 2 .

2. Coefficient Determination. The practical problem of determining the $C_{j}(r, s), D_{h}(r, s)$ for any given value of $\arg z$ is simplified by noticing that for $\nu$ fixed, it is sufficient to consider only certain diagonal sectors $S_{r, s}$.

Proposition 1. For $\nu \geqq 1$, let $k_{0}$ be the nonnegative integer such that 


$$
\nu-1 \leqq 4 k_{0} \leqq \nu+2, \text { or } 4 k_{0}-2 \leqq \nu \leqq 4 k_{0}+1
$$

Then

$$
\begin{array}{rll}
S_{r, r-k_{0}}: & \pi\left(\nu-\mu+2 r-3-2 k_{0}\right)<\arg z<\pi(\nu / 2-\mu+2 r) ; \\
& & \nu \geqq 2, k_{0} \geqq 1, \\
S_{r, r}: & \pi\left(2 r-\mu-\frac{3}{2}\right)<\arg z<\pi\left(2 r-\mu+\frac{1}{2}\right) ; & \nu=1, k_{0}=0, \\
S_{r, r-1}: \quad \pi\left(2 r-\mu-\frac{5}{2}\right)<\arg z<\pi\left(2 r-\mu-\frac{1}{2}\right) ; & \nu=1,
\end{array}
$$

and the diagonal sectors,

$$
\begin{array}{lll}
\nu=1 ; & S_{r, r}, S_{r, r-1}, & r=0, \pm 1, \pm 2, \cdots, \\
\nu \geqq 2 ; & S_{r, r-k_{0}}, & r=0, \pm 1, \pm 2, \cdots,
\end{array}
$$

completely cover the z-plane.

Thus, if the $(r, s)$ expansion is known, and $S_{r, 8}$ is one of the sectors in (2.3), it is sufficient to give recursion relations for the $(r \pm 1, s)$ and $(r, s \pm 1)$ expansions. These follow directly from the following $L_{i}$ and $G$ recursion relationships.

Proposition 2. Under the conditions of $(1.1), \nu \geqq k \geqq 1$, let the constants $C_{j}(k)$, $D_{h}(k)$ be chosen such that

$$
\begin{aligned}
& (-1)^{\nu+1}(2 \pi i)^{\nu} e^{i \pi\left(B_{1}-A_{1}\right)} e^{-i \pi t \nu} \frac{\Gamma\left(a_{P}-t\right) \Gamma\left(1-a_{P}+t\right)}{\Gamma\left(b_{Q}-t\right) \Gamma\left(1-b_{Q}+t\right)} \\
& =\sum_{i=1}^{p} C_{i}(k) e^{i \pi(1-2 k) t} \Gamma\left(a_{j}-t\right) \Gamma\left(1-a_{i}+t\right)-1+\sum_{h=1}^{\nu} D_{h}(k) e^{-i \pi 2 h t},
\end{aligned}
$$

an identity which can be built up from the case $k=1$, by repeated use of

$$
\begin{aligned}
\Gamma\left(a_{i}-t\right) \Gamma(1- & \left.a_{i}+t\right) \\
& =e^{i \pi 2 a_{j}} e^{-i \pi 2 t} \Gamma\left(a_{j}-t\right) \Gamma\left(1-a_{j}+t\right)+(-2 \pi i) e^{i \pi a_{j}} e^{-i \pi t} .
\end{aligned}
$$

In particular,

$$
\begin{array}{rlrl}
C_{i}(k) & =(-1)^{\nu+1}(2 \pi i)^{\nu} e^{i \pi\left(B_{1}-A_{1}\right)} e^{i \pi(2 k-\nu-1) a_{i}} \frac{\Gamma^{*}\left(a_{P}-a_{j}\right) \Gamma\left(1-a_{P}+a_{j}\right)}{\Gamma\left(b_{Q}-a_{i}\right) \Gamma\left(1-b_{Q}+a_{j}\right)}, \\
D_{h}(k) & =D_{h}(1)+(-2 \pi i) \sum_{j=1}^{p} C_{i}(1) e^{i \pi(2 h-1) a_{i}}, & 1 \leqq h \leqq k-1, \\
& =D_{h}(1), & k \leqq h \leqq \nu, \\
D_{\nu}(1) & =(-1)^{\nu+1} e^{i \pi 2\left(B_{1}-A_{1}\right)} . &
\end{array}
$$

Then,

$$
\begin{aligned}
& L_{i}(w)=e^{i \pi 2 a_{j}} L_{j}\left(w e^{-i \pi 2}\right)+(-2 \pi i) e^{i \pi a_{j}} G\left(w e^{-i \pi}\right), \\
& G(w)=\sum_{i=1}^{p} C_{i}(k) L_{i}\left(w e^{i \pi(1-2 k)}\right)+\sum_{h=1}^{\nu} D_{h}(k) G\left(w e^{-i \pi 2 h}\right) .
\end{aligned}
$$

Proof. The existence of the expansion (2.4) follows from the partial fraction decomposition 


$$
\begin{aligned}
& \frac{\prod_{i=1}^{q}\left(y-\beta_{i}\right)}{\prod_{i=1}^{p}\left(y-\alpha_{j}\right)}=\sum_{i=1}^{p} \frac{c_{j, k} y^{k}}{y-\alpha_{j}}+\sum_{h=0}^{\nu} d_{h, k} y^{h}, \\
& d_{\nu, k}=1 \quad d_{0, k}=(-1)^{\nu} \frac{\prod_{i=1}^{q} \beta_{i}}{\prod_{i=1}^{p} \alpha_{j}}, \\
& \quad \alpha_{j} \neq \alpha_{r}, \quad j \neq r, \quad 0<k \leqq \nu=q-p,
\end{aligned}
$$

with $y=e^{-i \pi 2 t}, \beta_{i}=e^{-i \pi 2 b_{i}}$ and $\alpha_{i}=e^{-i \pi 2 a_{i}}$. Multiplying (2.5), (2.4) by

$$
w^{t} \Gamma\left(b_{Q}-t\right)\left[\Gamma\left(a_{P}-t\right)\right]^{-1},
$$

and integrating along a contour $L_{+}$which separates the poles of $\Gamma\left(b_{Q}-t\right)$ from those of $\Gamma\left(1-a_{P}+t\right)$, we arrive at $(2.7)$ and an expansion of $G_{p, q}^{0, p}\left(w e^{-i \pi \nu}\right) \equiv 0$ which reduces to (2.8), respectively.

Remark 1. Equations (2.7) and (2.8) can also be written in the form

$$
\begin{aligned}
L_{j}(w) & =e^{-i \pi 2 a_{i}} L_{j}\left(w e^{i \pi 2}\right)+(2 \pi i) e^{-i \pi a_{j}} G\left(w e^{i \pi}\right), \\
-D_{\nu}(1) G\left(w e^{-\pi 2 \nu}\right) & =\sum_{i=1}^{p} C_{i}(k) L_{i}\left(w e^{i \pi(1-2 k)}\right)+\sum_{h=1}^{\nu} D_{h-1}(k) G\left(w e^{i \pi(2-2 h)}\right), \\
1 & \leqq k \leqq \nu, \quad D_{0}(k)=-1 .
\end{aligned}
$$

Using Proposition 2, the variables of $L_{j}(w)$ and $G(w)$ can be changed in a systematic fashion.

Proposition 3. Under the conditions (1.1), let $k=r-s, 1 \leqq k+1 \leqq \nu$. Then the following recursion relations hold.

$\underline{(r, s) \rightarrow(r+1, s)}$

$$
\begin{aligned}
C_{j}(r+1, s) & =e^{i \pi 2 a_{i}} C_{j}(r, s), \\
D_{h}(r+1, s) & =D_{h}(r, s), \quad h \neq k+1, \\
& =D_{k+1}(r, s)+(-2 \pi i) \sum_{j=1}^{p} C_{i}(r, s) e^{i \pi a_{j}}, \quad h=k+1,
\end{aligned}
$$

$\underline{(r+1, s) \rightarrow(r+1, s+1)}$

$$
\begin{aligned}
C_{j}(r+1, s+1) & =C_{i}(r+1, s)+D_{1}(r+1, s) C_{j}(k+1), \\
D_{h}(r+1, s+1) & =D_{1}(r+1, s) D_{h}(k+1)+D_{h+1}(r+1, s), \\
& =D_{1}(r+1, s) D_{\nu}(k+1), \quad h \leqq h \leqq \nu-1,
\end{aligned}
$$

$\underline{(r, s) \rightarrow(r, s-1)}$

$$
\begin{aligned}
C_{i}(r, s-1) & =C_{j}(r, s)-\frac{D_{\nu}(r, s)}{D_{\nu}(1)} C_{j}(k+1), \\
D_{h}(r, s-1) & =\frac{D_{\nu}(r, s)}{D_{\nu}(1)}, \quad h=1, \\
& =D_{h-1}(r, s)-\frac{D_{\nu}(r, s)}{D_{\nu}(1)} D_{h-1}(k+1), \quad 2 \leqq h \leqq \nu,
\end{aligned}
$$


$\underline{(r, s-1) \rightarrow(r-1, s-1)}$

$$
\begin{aligned}
C_{j}(r-1, s-1) & =e^{-i \pi 2 a_{i}} C_{j}(r, s-1), \\
D_{h}(r-1, s-1) & =D_{h}(r, s-1), \quad h \neq k+1, \\
& =D_{k+1}(r, s-1)+(2 \pi i) \sum_{j=1}^{p} C_{j}(r, s-1) e^{-i \pi a_{i}}
\end{aligned}
$$

These recursion formulae are valid if $k=k_{0}$.

Proof. Equations (2.12) and (2.15) follow from (2.7) and (2.10), respectively, whereas Eqs. (2.13) and (2.14) follow when the $h=1$ and $h=\nu$ terms in the $(r+1, s)$ or $(r, s)$ expansions are replaced by the expansions given in (2.8) and (2.11), respectively.

Suppose that an $(s, s)$ expansion for $G_{p, e}^{m, n}(z)$ is known, $s$ arbitrary. Then $k_{0}$ applications of (2.12) yield the $\left(s+k_{0}, s\right)$ expansion for $G_{p, a}^{m, n}(z)$, and Propositions 1,3 imply the asymptotic behaviour of $G_{p, q}^{m, n}(z)$ for all values of $\arg z$ as $z \rightarrow \infty$. Hence, the only remaining practical problem is to find a particular $(s, s)$ expansion.

Proposition 4. Under the conditions of (1.1), $\nu \geqq 1$, there exist constants $E_{i}, F_{h}$ and $H_{j}$ such that

$$
\frac{\Gamma\left(a_{N}-t\right) \Gamma\left(1-a_{N}+t\right)}{\Gamma_{m}\left(b_{Q}-t\right) \Gamma_{m}\left(1-b_{Q}+t\right)}-\frac{\Gamma\left(a_{P}-t\right) \Gamma\left(1-a_{P}+t\right)}{\Gamma\left(b_{Q}-t\right) \Gamma\left(1-b_{Q}+t\right)} \sum_{j=0}^{\mu-\nu} H_{j} e^{i \pi(\mu-\nu-2 j) t}
$$

$$
\begin{aligned}
= & \sum_{j=1}^{p} E_{j} e^{i \pi(\mu+1-2 s) t} \Gamma\left(a_{i}-t\right) \Gamma\left(1-a_{j}+t\right) \\
& +\sum_{h=1}^{\nu} F_{h} e^{i \pi(\mu+2-2 s-2 h) t},
\end{aligned}
$$

$s=\max (0,1+\mu-\nu)$. Then,

$$
E_{i}=C_{i}(s, s), \quad F_{h}=D_{h}(s, s) .
$$

In particular, if $\mu \leqq \nu-1,(2.16)$ reduces to

$$
\begin{aligned}
& \frac{\Gamma\left(a_{N}-t\right) \Gamma\left(1-a_{N}+t\right)}{\Gamma_{m}\left(b_{Q}-t\right) \Gamma_{m}\left(1-b_{Q}+t\right)} \\
& =\sum_{i=1}^{n} C_{i}(0,0) e^{i \pi(\mu+1) t} \Gamma\left(a_{i}-t\right) \Gamma\left(1-a_{i}+t\right) \\
& \quad+\sum_{h=1}^{1+\mu} D_{h}(0,0) e^{i \pi(\mu+2-2 h) t}, \\
& C_{i}(0,0)=e^{-i \pi(\mu+1) a_{i}} \frac{\Gamma\left(1+a_{j}-a_{N}\right) \Gamma^{*}\left(a_{N}-a_{j}\right)}{\Gamma_{m}\left(1-b_{Q}+a_{j}\right) \Gamma_{m}\left(b_{0}-a_{i}\right)}, \quad j=1, \cdots, n .
\end{aligned}
$$

If $\mu \leqq-1$, the sum $\sum_{h=1}^{1+\mu}$ in (2.18) also disappears.

Proof. We begin by noticing that (2.17) is a direct consequence of (2.16). For if (2.16) is multiplied by $z^{t} \Gamma\left(b_{Q}-t\right)\left[\Gamma\left(a_{P}-t\right)\right]^{-1}$, and the resulting expansion is integrated over the contour $L_{+}$of (1.3), one obtains the $(s, s)$ expansion of

$$
G_{p, q}^{m, n}(z)-\sum_{j=0}^{\mu-\nu} H_{j} G_{p, q}^{0, p}\left(z e^{i \pi(\mu-\nu-2 j)}\right)=G_{p, Q}^{m, n}(z) .
$$


First, assume that $\mu \leqq \nu-1$. Then (2.18) follows directly from the partial fraction decomposition

$$
\frac{\prod_{i=m+1}^{a}\left(y-\beta_{i}\right)}{\prod_{i=1}^{n}\left(y-\alpha_{i}\right)}=\sum_{j=1}^{n} \frac{e_{j}}{y-\alpha_{i}}+\sum_{h=0}^{\mu} f_{h} y^{h},
$$

with $y=e^{-i \pi 2 t}, \beta_{j}=e^{-i \pi 2 b_{j}}$ and $\alpha_{i}=e^{-i \pi 2 a_{j}}$. Similarly for the case, $\mu \geqq \nu$, we prove by induction on $s, 0 \leqq s \leqq 1+\mu-\nu$, that there exist constants $e_{i, s}, f_{h, s}$ and $h_{i, 8}$ such that

$$
\begin{aligned}
\frac{\prod_{i=m+1}^{a}\left(y-\beta_{i}\right)}{\prod_{i=1}^{n}\left(y-\alpha_{j}\right)}- & \left(\sum_{j=0}^{s-1} h_{i, s} y^{i}\right) \frac{\prod_{i=1}^{a}\left(y-\beta_{j}\right)}{\prod_{i=1}^{p}\left(y-\alpha_{i}\right)} \\
& =\sum_{j=1}^{p} \frac{e_{i, s} y^{s}}{y-\alpha_{j}}+\sum_{h=s}^{\mu} f_{h, s} y^{h}, \quad 0 \leqq s \leqq 1+\mu-\nu .
\end{aligned}
$$

If $s=0$, (2.21) reduces to (2.20). Assuming (2.21) valid for a particular value of $s \leqq \mu-\nu$, we note that systematic application of

$$
1 /(y-a)=y / a(y-a)-1 / a,
$$

allows us to write $R(y)$, the left-hand side of (2.21), in the form

$$
R(y)=\sum_{i=1}^{p} \frac{e_{i, s} y^{s+1}}{\alpha_{j}\left(y-\alpha_{j}\right)}+y^{s}\left\{f_{s, s}-\sum_{j=1}^{p} \frac{e_{i, s}}{\alpha_{j}}\right\}+\sum_{h=s+1}^{\mu} f_{h, s} y^{h} .
$$

Finally, if the expansion (2.9) with $k=1$ is multiplied by $y^{s} h_{s, s}$,

$$
d_{0,1} h_{s, s}=f_{s, s}-\sum_{j=1}^{p}\left(e_{i, s} / \alpha_{i}\right)
$$

and the resulting expression is subtracted from (2.23), one obtains (2.21) with $s$ replaced by $s+1$. Equation (2.16) then follows from (2.21) with $s=1+\mu-\nu$, $y=e^{-i \pi 2 t}, \beta_{i}=e^{-i \pi 2 b_{i}}$ and $\alpha_{i}=e^{-i \pi 2 a_{i}}$.

Remark 2. A particular $(r, s)$ expansion may directly imply the asymptotic expansion of $G_{p, q}^{m, n}(z)$ in a larger sector than $S_{r, s}$. For example, if $\nu \geqq 1, \mu \leqq-1$, it follows from Proposition 4 that

$$
G_{p, q}^{m, n}(z)=\sum_{j=1}^{n} C_{j}(0,0) L_{i}\left(z e^{i \pi(\mu+1)}\right),
$$

an expansion which, though valid for all values of $\arg z$, directly implies the asymptotic behaviour of $G_{p, q}^{m, n}(z)$ only when $-\pi(\nu / 2+\mu+2)<\arg z<\pi(\nu / 2-\mu)$.

Remark 3. Once a particular $(r, s)$ expansion has been derived, the conditions (1.1) can be weakened by an appeal to analytic continuation.

Remark 4. The above results are easily transformed into results for the hypergeometric functions,

$$
{ }_{p} F_{Q}\left(\begin{array}{c}
\alpha_{P} \\
\beta_{Q}
\end{array} \mid-z\right)=\frac{\Gamma\left(\beta_{Q}\right)}{\Gamma\left(\alpha_{P}\right)} G_{p, Q+1}^{1, p}\left(z \mid \begin{array}{l}
1-\alpha_{P} \\
0,1-\beta_{Q}
\end{array}\right) .
$$

3. Generalizations. The above expansions are derived by multiplying certain identities by the function $z^{t} \Gamma\left(b_{Q}-t\right)\left[\Gamma\left(a_{P}-t\right)\right]^{-1}$ and integrating the resultant 
identities over an appropriate contour. If these identities are multiplied by more general functions, the above expansions can be generalized.

Let $c_{i}, j=1, \cdots, k ; d_{i}, j=1, \cdots, v ; f_{i}, j=1, \cdots, w$, be constants such that

$$
c_{j}-b_{u} \neq \text { a positive integer; } j=1, \cdots, k ; u=1, \cdots, q .
$$

Then Theorem 3 can be generalized as follows.

THEOREM 4. Let $a_{i}, b_{i}, c_{i}$ satisfy the conditions (1.1) and (3.1). If the expansion (1.12) is derivable from Propositions 2, 3 and 4, then

$$
\begin{aligned}
G_{p+k+v, q+w}^{m, n+k}\left(z \mid \begin{array}{l}
c_{K}, a_{P}, d_{V} \\
b_{Q}, f_{W}
\end{array}\right) & \\
= & \sum_{i=1}^{p} C_{i}(r, s) G_{p+k+v, q+w}^{a, k+1}\left(z e^{i \pi(\mu+1-2 r)} \mid \begin{array}{l}
c_{K}, a_{j}, a_{P}^{* j}, d_{V} \\
b_{Q}, f_{W}
\end{array}\right) \\
& +\sum_{h=1}^{\nu} D_{h}(r, s) G_{p+k+v, q+w}^{a, k}\left(z e^{i \pi(\mu+2-2 s-2 h)} \mid \begin{array}{l}
c_{K}, a_{P}, d_{V} \\
b_{Q}, f_{W}
\end{array}\right),
\end{aligned}
$$

where $a_{P}^{* i}$ denotes the sequence $a_{1}, \cdots, a_{p}$ with $a_{j}$ deleted, and $\nu, \mu, C_{i}(r, s), D_{h}(r, s)$ have the same values as in (1.12).

Proof. If the expansion (1.12) can be built up from Propositions 2, 3 and 4, the expansion (3.2) can be built up from appropriate generalizations of (2.7), (2.8) and (2.19). In particular, if (2.5) and (2.4) are multiplied by

$$
z^{t} \Gamma\left(b_{Q}-t\right) \Gamma\left(1-c_{K}+t\right)\left[\Gamma\left(a_{P}-t\right) \Gamma\left(d_{V}-t\right) \Gamma\left(1-f_{W}+t\right)\right]^{-1},
$$

and integrated over a contour $L_{+}^{*}$ which separates the poles of $\Gamma\left(b_{Q}-t\right)$ from those of $\Gamma\left(1-a_{P}+t\right) \Gamma\left(1-c_{K}+t\right)$, one obtains

$$
\begin{aligned}
& L_{j}^{*}(z)=e^{i \pi 2 a_{i}} L_{j}^{*}\left(z e^{-i \pi 2}\right)+(-2 \pi i) e^{i \pi a_{i}} G^{*}\left(z e^{-i \pi}\right), \\
& G^{*}(z)=\sum_{j=1}^{p} C_{j}(u) L_{i}^{*}\left(z e^{i \pi(1-2 u)}\right)+\sum_{h=1}^{\nu} D_{h}(u) G^{*}\left(z e^{-i \pi 2 h}\right),
\end{aligned}
$$

where $1 \leqq u \leqq \nu$, and

$$
\begin{aligned}
& G^{*}(z)=G_{p+k+v, q+w}^{q, k}\left(z \mid \begin{array}{l}
c_{K}, a_{P}, d_{V} \\
b_{Q}, f_{W}
\end{array}\right), \\
& L_{j}^{*}(z)=G_{p+k+v, q+w}^{a, k+1}\left(z \mid \begin{array}{l}
c_{K}, a_{i}, a_{P}^{* j}, d_{V} \\
b_{Q}, f_{W}
\end{array}\right) .
\end{aligned}
$$

Treating (2.16) similarly, one obtains (3.2) with $r=s$.

The significance of (3.2) lies in the fact that if $c_{K}, d_{V}$ and $f_{W}$ are "large", then in some restricted sense and in some restricted region,

$$
\begin{aligned}
& C G^{*}(z) \quad \text { behaves like } G(\Omega z), \\
& C L_{i}^{*}(z) \text { behaves like } L_{i}(\Omega z), \\
& C=\frac{\Gamma\left(d_{V}\right) \Gamma\left(1-f_{W}\right)}{\Gamma\left(1-c_{K}\right)}, \quad \Omega=\prod_{i=1}^{v} d_{i} \prod_{i=1}^{k}\left(1-c_{i}\right) / \prod_{i=1}^{w}\left(1-f_{i}\right) .
\end{aligned}
$$

A special $k=1, w=0, v=1$ case of Theorem 4 is discussed in [4]. 
Department of Mathematics

The University of Alberta

Edmonton 7, Alberta, Canada

1. C. S. MeiJer, "On the G-function. I-VIII," Nederl. Akad. Wetensch. Proc. Ser. A, v. 49,1946 , pp. $227-237,344-356,457-469,632-641,765-772,936-943,1063-1072,1165-$ $1175=$ Indag. Math., v. 8, 1946, pp. 124-134, 213-225, 312-324, 391-400, 468-475, 595-602, 661-670, 713-723. MR 8, 156; MR 8, 379.

2. Y. L. LUKE, The Special Functions and Their Approximations. Vols. I, II, Math. in Sci. and Engineering, Vol. 53, Academic Press, New York, 1969. MR 39 \#3039; MR 40 \#2909.

3. E. W. BARNES, "The asymptotic expansion of integral functions defined by generalized hypergeometric series," Proc. London Math. Soc. (2), v. 5, 1907, pp. 59-116.

4. J. L. FIELDS, "A linear scheme for rational approximations," J. Approximation Theory, v. 6, 1972. (To appear.) 